\title{
Correction to: Post-migration Social-Environmental Factors Associated with Mental Health Problems among Asylum Seekers: A Systematic Review
}

\author{
Sohail Jannesari ${ }^{1}\left[\right.$. Stephani Hatch ${ }^{2,3} \cdot$ Matthew Prina $^{1} \cdot$ Sian Oram ${ }^{1}$
}

Published online: 21 June 2020

(c) The Author(s) 2020

\section{Correction to: Journal of Immigrant and Minority Health https://doi.org/10.1007/s10903-020-01025-2}

The original version of the article unfortunately contained small errors in Table 1 and the text under Result section. These errors do not affect the paper's main findings in any way.

The below listed are the errors in Table 1.

- Footnote 'a' should be deleted and Column 6 renamed 'Primary countries or regions of origin'. Instead of 'Not Available', the country/region column should say Africa for Wong et al.'s paper. Pakistan has been added as a primary country for Hocking et al.

- The PTSD tool for Kaltenbach et al. is PSS-I/PCL-5 not the HTQ as reported before. The study country in Kashyap et al. is the USA not Australia and the PTSD tool is the HTQ not PCL. Heeren et al. used the HSCL-25 and PDS in addition to the MINI.

- Whitsett et al. conducted a prospective cohort study, not a cross-sectional study as reported. Boersma used a casecontrol design.

The correct Table 1 is presented here.

In the second paragraph under the 'Results' section, the sentence 'with the USA (19\% of studies) and Australia (19\%) most frequent.' should be read as 'with the USA (24\% of studies) most frequent.' and the sentence ' $71 \%$ of studies were cross-sectional' should read ' $67 \%$ of studies were cross-sectional'.

1 Health Service and Population Research, The Institute of Psychiatry, Psychology and Neuroscience, David Goldberg Building, IoPPN, 16 De Crespigny Park, London SE5 8AF, UK

2 Psychological Medicine, The Institute of Psychiatry, Psychology and Neuroscience, King's College London, London, UK

3 ESRC Centre for Society and Mental Health, King's College London, London, UK 
Table 1 Study characteristics [15, 20, 33-51]

\begin{tabular}{|c|c|c|c|c|c|c|c|c|c|c|}
\hline \multirow[t]{2}{*}{ Lead author } & \multirow[t]{2}{*}{ Year } & \multirow[t]{2}{*}{$\mathrm{N}$} & \multicolumn{2}{|c|}{ Gender } & \multirow[t]{2}{*}{ Age $(\bar{x})$} & \multirow{2}{*}{$\begin{array}{l}\text { Primary countries or } \\
\text { regions of origin }\end{array}$} & \multirow[t]{2}{*}{ Host } & \multirow[t]{2}{*}{ Design } & \multirow[t]{2}{*}{ Outcomes } & \multirow[t]{2}{*}{ Tool } \\
\hline & & & $\mathrm{M}$ & $\mathrm{F}$ & & & & & & \\
\hline Boersma & 2005 & 117 & 70 & 47 & 41.6 & Nigeria, Lebanon & USA & Case-control & $\begin{array}{l}\text { Depression } \\
\text { Somatoform }\end{array}$ & $\begin{array}{l}\text { HSCL-25 } \\
\text { SCL-90 }\end{array}$ \\
\hline Eisen & 2016 & 78 & 33 & 45 & 34.1 & Ethiopia, Cameroon & USA & Prospect. cohort & $\begin{array}{l}\text { Depression } \\
\text { PTSD }\end{array}$ & $\begin{array}{l}\text { HSCL-25 } \\
\text { HTQ-30 }\end{array}$ \\
\hline Hecker & 2018 & 61 & 56 & 5 & 28.64 & Afghanistan, Syria & Switzer. & $\mathrm{CS}$ & $\begin{array}{l}\text { Depression } \\
\text { PTSD, CPTSD }\end{array}$ & $\begin{array}{l}\text { PHQ-9 } \\
\text { ICD-11 }\end{array}$ \\
\hline Heeren & 2012 & 86 & 60 & 16 & 29.8 & $\begin{array}{l}\text { Africa and the } \\
\text { Middle East }\end{array}$ & Switzer. & $\mathrm{CS}$ & $\begin{array}{l}\text { Depression } \\
\text { Anxiety } \\
\text { PTSD }\end{array}$ & $\begin{array}{l}\text { MINI/ } \\
\text { HSCL-25 } \\
\text { PDS }\end{array}$ \\
\hline Hocking & 2015 & 115 & 103 & 102 & 35.2 & Sri Lanka, Pakistan & Australia & $\mathrm{CS}$ & $\begin{array}{l}\text { Depression } \\
\text { Anxiety } \\
\text { PTSD }\end{array}$ & $\begin{array}{l}\text { HCSL-25 } \\
\text { HSCL-25 } \\
\text { HTQ }\end{array}$ \\
\hline Kaltenbach & 2018 & 15 & 4 & 11 & 35.87 & Syria, Iraq, Iran & Germany & Prospect. cohort & $\begin{array}{l}\text { Depression } \\
\text { PTSD }\end{array}$ & $\begin{array}{l}\text { PHQ-9 } \\
\text { PSS-I/PCL-5 }\end{array}$ \\
\hline Kashyap & 2019 & 122 & 78 & 44 & 39.07 & & USA & Prospect. cohort & $\begin{array}{l}\text { Depression } \\
\text { PTSD }\end{array}$ & $\begin{array}{l}\text { PHQ-9 } \\
\text { HTQ }\end{array}$ \\
\hline Laban & 2005 & 294 & 190 & 104 & & Iraq & Netherlands & CS (Control) & $\begin{array}{l}\text { Depression } \\
\text { Anxiety, PTSD, } \\
\text { Somatoform }\end{array}$ & CIDI \\
\hline Morgan & 2017 & 42 & & & & $\begin{array}{l}\text { African countries Inc. } \\
\text { Zimbabwe, DRC/ } \\
\text { Congo }\end{array}$ & UK & $\mathrm{CS}$ & $\begin{array}{l}\text { Depression } \\
\text { Anxiety } \\
\text { PTSD }\end{array}$ & $\begin{array}{l}\text { HCSL-25 } \\
\text { HSCL-25 } \\
\text { HTQ }\end{array}$ \\
\hline Müller & 2018 & 78 & 33 & 45 & 38.2 & Turkey & Germany & $\mathrm{CS}$ & $\begin{array}{l}\text { Depression } \\
\text { Anxiety, PTSD } \\
\text { Schizophrenia }\end{array}$ & ICD-10 \\
\hline Nakash & 2017 & 90 & 90 & 0 & 30.7 & Sudan, Eritrea & Israel & $\mathrm{CS}$ & $\begin{array}{l}\text { Depression } \\
\text { Anxiety } \\
\text { PTSD }\end{array}$ & $\begin{array}{l}\text { HSCL-25 } \\
\text { HSCL-25 } \\
\text { PCL }\end{array}$ \\
\hline Nickerson & 2015 & 30 & 23 & 7 & & Turkey & Switzer. & $\mathrm{CS}$ & $\begin{array}{l}\text { Depression } \\
\text { PTSD }\end{array}$ & $\begin{array}{l}\text { HSCL-25 } \\
\text { PDS }\end{array}$ \\
\hline Ryan & 2008 & 162 & 202 & 152 & 32.5 & Nigeria & Ireland & Prospect. cohort & Distress & SCL-90R \\
\hline Schock & 2015 & 50 & 30 & 20 & 32.1 & Iran, Turkey, Balkans & Germany & Prospect. cohort & $\begin{array}{l}\text { Depression } \\
\text { Anxiety } \\
\text { PTSD }\end{array}$ & $\begin{array}{l}\text { HCSL-25 } \\
\text { HCSL-25 } \\
\text { PDS }\end{array}$ \\
\hline Silove & 1997 & 40 & 21 & 19 & 35 & & Australia & $\mathrm{CS}$ & $\begin{array}{l}\text { Depression } \\
\text { Anxiety } \\
\text { PTSD }\end{array}$ & $\begin{array}{l}\text { HCSL-25 } \\
\text { HCSL-25 } \\
\text { CIDI }\end{array}$ \\
\hline Slonim-Nevo & 2015 & 340 & 276 & 64 & 30.6 & Sudan & Israel & $\mathrm{CS}$ & PTSD & PCL \\
\hline Sohn & 2019 & 129 & 93 & 36 & & Nigeria, Ethiopia & S. Korea & $\mathrm{CS}$ & $\begin{array}{l}\text { Depression } \\
\text { PTSD }\end{array}$ & $\begin{array}{l}\text { PHQ-9 } \\
\text { IES-R }\end{array}$ \\
\hline Song & 2010 & 44 & 24 & 20 & 36 & Iran, Eritrea, Iraq & USA & $\mathrm{CS}$ & $\begin{array}{l}\text { Depression } \\
\text { Anxiety } \\
\text { PTSD }\end{array}$ & $\begin{array}{l}\text { HSCL-25 } \\
\text { HSCL-25 } \\
\text { PCL }\end{array}$ \\
\hline Steel & 1999 & 296 & 135 & 64 & 43.7 & Sri Lanka & Australia & $\mathrm{CS}$ & PTSD & HTQ \\
\hline Whitsett & 2017 & 105 & 41 & 64 & 34.76 & Ethiopia, Cameroon & USA & Prospect. cohort & $\begin{array}{l}\text { Depression } \\
\text { Anxiety } \\
\text { PTSD }\end{array}$ & $\begin{array}{l}\text { HCSL-25 } \\
\text { HSCL-25 } \\
\text { HTQ }\end{array}$ \\
\hline Wong & 2016 & 374 & 292 & 82 & 31.52 & Africa & China-HK & $\mathrm{CS}$ & PTSD & PHQ-2 \\
\hline
\end{tabular}

Publisher's Note Springer Nature remains neutral with regard to jurisdictional claims in published maps and institutional affiliations. 